\title{
Análise Comparativa de Ferramentas CAD e BIM para Projetos de Instalações Elétricas
}

\section{A Comparative Analysis Between CAD and BIM Tools for Electrical Projects}

\section{Cibelly Cristina Rodrigues Couto}

Universidade Federal de Uberlândia (UFU) | Uberlândia | Brasil |

cibellycristinaudi@gmail.com

\section{Leonardo Nery Carrijo dos Santos}

Universidade Federal de Uberlândia (UFU) | Uberlândia | Brasil |

leonardoncsantos@gmail.com

\section{Edgard Afonso Lamounier Júnior}

Universidade Federal de Uberlândia (UFU) |Uberlândia | Brasil | elamounier@gmail.com

\section{Gerson Flavio Mendes de Lima}

Universidade Federal de Uberlândia (UFU) | Uberlândia | Brasil |

gersonlima@ieee.org

\section{Alexandre Cardoso}

Universidade Federal de Uberlândia (UFU) | Uberlândia | Brasil |

alexandre@ufu.br

\section{Resumo}

No campo da arquitetura e da engenharia, os desenhos de projetos fornecem informações relevantes sobre a obra. $O$ surgimento do sistema CAD alterou diversos ramos da indústria de projetos e até hoje é um dos principais métodos utilizados por profissionais da área. Posteriormente, o BIM surgiu como um conceito promissor por possibilitar melhorias gráficas ao mesmo tempo que integra os participantes do projeto nas diversas fases da obra. No presente artigo, um comparativo é realizado entre as metodologias CAD e BIM por intermédio de um projeto de instalações elétricas residencial elaborado pela empresa CGWorks.

Palavras-chave: AutoCAD 2D. Revit. Instalações Elétricas. CAD. BIM.

\begin{abstract}
In the field of architecture and engineering, design drawings provide relevant information about the work. The emergence of the Computer-Aided Design (CAD) system changed several branches of the design industry and to this day is one of the main methods used by professionals in the field. Subsequently, Building Information Modeling (BIM) emerged as a promising concept for enabling graphical improvements while integrating the project participants in the various
\end{abstract}

Como citar:

COUTO, C. C. R. .; SANTOS , L. N. C. dos .; LAMOUNIER JÚNIOR , E. A. .; LIMA, G. F. M. de; CARDOSO, A. . Análise comparativa de ferramentas CAD e BIM para projetos de instalações elétricas. In: SIMPÓSIO BRASILEIRO DE TECNOLOGIA DA INFORMAÇÃO E COMUNICAÇÃO NA CONSTRUÇÃO, 3., 2021, Uberlândia. Anais [...]. Porto Alegre: ANTAC, 2021. p. 1-7. Disponível em:

https://eventos.antac.org.br/index.php/sbtic/article/view/629. Acesso em: 3 ago. 2021.p.1-8. 
phases of the work. In this paper, a comparison is made between CAD and BIM methodologies through a residential electrical installations project prepared by CGWorks.

Keywords: AutoCAD 2D. Revit. Electrical Installations. CAD. BIM.

\section{INTRODUÇÃO}

Projetos realizados pela tecnologia CAD - Computer-Aided Design (Desenho Assistido por Computador) foram introduzidos na indústria da Arquitetura, Engenharia e Construção (AEC) a partir da década de 1980, a fim de digitalizar ativos e tornar tal processo de concepção mais eficiente [1].

Entretanto, a tecnologia CAD, quando empregada, apresenta limitações no que tange a comunicação entre projetistas, bem como uma visualização abrangente do sistema, uma vez que tais plataformas normalmente não apresentam uma troca de dados síncrona, nem mesmo um ambiente de trabalho integrado [1]. Assim, podem ser gerados conflitos de informação entre os usuários, de tal forma que $30 \%$ do valor total do projeto seja desperdiçado, como aponta [2]. Dessa forma, surgiu a metodologia de criação de modelos virtuais BIM - Building Information Modeling (Modelagem da Informação na Construção) objetivando reduzir tais impactos uma vez que, além da modelagem tridimensional, esta permite a geração de objetos parametrizados com atributos associados em uma plataforma colaborativa de disciplinas compatibilizadas [1].

Como aponta [3], vale ressaltar que alguns países já são exemplos na adoção dessa metodologia para o setor de construções, como Reino Unido e Singapura, ao passo que outros como Estados Unidos e Holanda já exigem BIM em projetos custeados pelo governo. Analogamente, o Governo Federal do Brasil editou em 02 de abril de 2020 o Decreto no 10.306/2020, o qual torna imperativo a utilização do BIM em projetos de arquitetura e engenharia em obras e serviços públicos federais iniciadas em 2021 [4], de tal forma a difundir a tecnologia BIM nos setores de natureza pública e privada. No que concerne à área de instalações elétricas, a metodologia BIM é, possivelmente, pouco aplicada, como sugere [5].

Objetiva-se, portanto, por meio deste trabalho demonstrar os benefícios e desafios que a metodologia BIM pode apresentar na área de instalações elétricas. Para tal, o primeiro tópico contempla a definição teórica das metodologias CAD e BIM, juntamente com suas características na compatibilização de projetos. Posteriormente, é apresentado um estudo de caso acerca de um projeto elétrico residencial realizado em ferramentas do tipo CAD e BIM, sendo feito, portanto, um comparativo entre ambas. Por fim, são apresentadas algumas considerações feitas durante tal comparação bem como possíveis trabalhos futuros a serem contemplados.

\section{METODOLOGIAS DE REPRESENTAÇÃO E MODELAGEM DE PROJETOS}

Como observado em [6] e [7], nas últimas décadas, a indústria da AEC vem sendo modificada, uma vez que os processos de execução de obras estão cada vez mais 
complexos e interligados, de tal forma que sejam necessárias ferramentas mais robustas e inteligentes no decorrer do projeto. Nesse sentido, como observado em [7], começaram a surgir tecnologias que vinculassem os artefatos digitais de uma construção em um ambiente colaborativo de representação e visualização de dados. Tal metodologia é, normalmente, vinculada ao acrônimo BIM.

\section{TECNOLOGIA CAD}

Observa-se em [8] o processo gradativo de substituição dos métodos e artefatos convencionais de elaboração de projetos, pela tecnologia CAD, possibilitando aos projetistas a digitalização dos ativos referentes às plantas em esquemas bi ou tridimensionais. Assim, considera-se que a indústria da AEC obteve um ganho relevante em termos de flexibilidade, otimização e precisão [7][8].

Entretanto, vale ressaltar, como sugere [8], que a engenharia contemporânea é, tradicionalmente, norteada pela análise de dados e comunicação entre sistemas. Nesse sentido, observa-se que, ainda que o CAD tenha aprimorado os sistemas de visualização e representação de projetos e de modelagem de equipamentos, não houve, possivelmente, uma mudança de paradigma no que tange ao fluxo de trabalho.

Ressalta-se na Figura 1, por exemplo, que a execução de tarefas em plataformas CAD ocorre de forma linear e individualizada, o que pode, por conseguinte, resultar em realimentações em outras disciplinas de execução e, assim, tornar o projeto mais oneroso e custoso. Ademais, identifica-se, normalmente, que tal fluxo de trabalho dificulta a integração entre projetistas além de possibilitar o aumento da probabilidade de erros, pois cada disciplina é realizada em plataformas independentes as quais lidam com uma grande quantidade de dados a serem verificados a cada atualização e processo de compatibilização do projeto.

Figura 1: Fluxo de trabalho de um projeto CAD

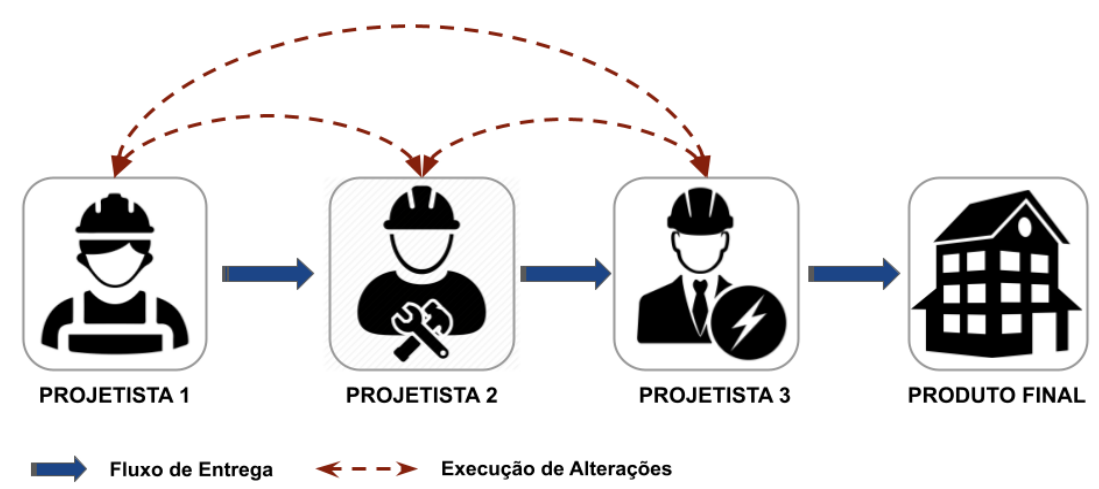

Fonte: o autor.

\section{METODOLOGIA BIM}

Desenvolvida ao longo dos últimos anos, a metodologia BIM propõe uma modelagem multidimensional, ou seja, que reúne tanto dados quantitativos quanto qualitativos de ativos em uma mesma solução, permitindo estimativas de tempo e custo, detecção de conflitos e tomadas de decisão em um ambiente de trabalho mais integrado e colaborativo [8]. 
Dessa forma, a aplicação da metodologia BIM na indústria da AEC pode representar uma mudança de paradigma e também de fluxo de trabalho visto que o projeto é desenvolvido de forma coordenada em uma única plataforma, como o software Autodesk Revit, na qual as diferentes disciplinas de uma edificação são desenvolvidas separadamente, porém integradas na solução final, como apontam [7][8].

Para tal, o projeto em BIM garante a compatibilidade entre os diversos projetistas como requisito inicial de execução, e não como um processo realizado a cada mudança de esfera de trabalho, como é mostrado na Figura 1, na abordagem em CAD. Portanto, o ambiente de trabalho em BIM permite a troca de informações entre os projetistas em tempo real bem como a execução rápida e coordenada das tarefas de cada disciplina, como é visto na Figura 2.

Figura 2: Fluxo de trabalho de um projeto BIM

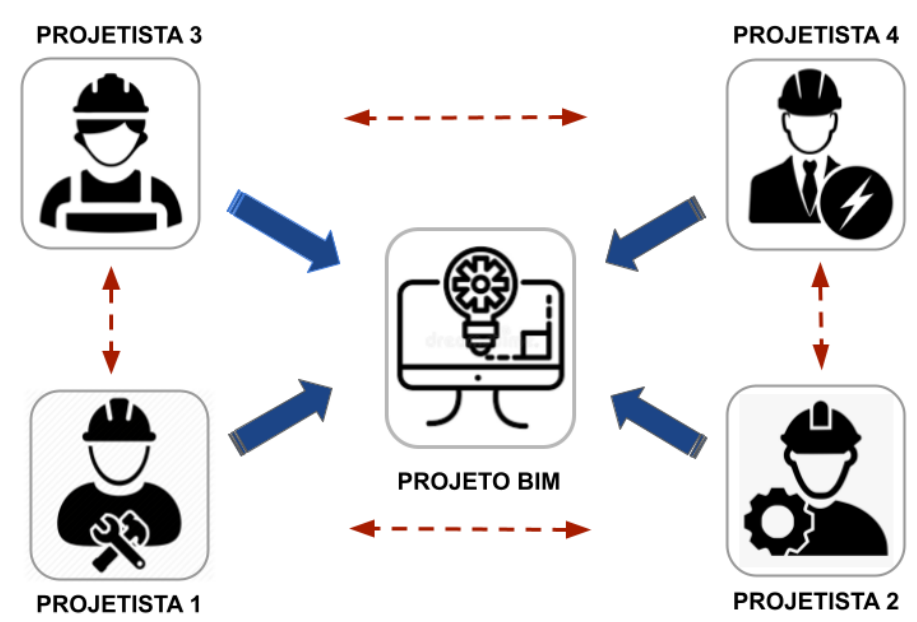

Fonte: o autor.

\section{ESTUDO DE CASO}

Apesar das diversas evoluções tecnológicas ocorridas no campo de edificações, ainda hoje existe uma forte tradição da utilização de ferramentas CAD na elaboração de projetos de instalações elétricas [5], pois, uma vez que os processos estão bem definidos, muitas empresas optam por não adotar a metodologia BIM por questões financeiras e até mesmo por falta de qualificação, como sugere [5].

Contudo, explorar as ferramentas BIM pode resultar em reduções consideráveis de custo. De acordo com [9][10], projetos de edificações apresentam um desperdício médio entre 5 a 10\%, bem como prejuízos referentes a mudanças orçamentárias não previstas, o que poderia ser reduzido com a utilização de BIM. Tal fato decorre da capacidade dessa metodologia de promover a interoperabilidade de ferramentas, ou seja, a capacidade de intercambiar dados e ativos entre softwares bem como visualizar tridimensionalmente diversos aspectos do projeto. Dessa maneira, pode-se detectar, ainda no ambiente virtual, impasses na compatibilização do projeto de instalações em relação às disciplinas arquitetônica e estrutural. Assim, podem ser aplicadas ações preventivas mediante as possíveis falhas na execução. 
Para o estudo de caso, utilizou-se o projeto elétrico de uma residência elaborado pela empresa CGW nos softwares AutoCAD 2D, apoiado pelo módulo de extensão Visual Electric, que foi desenvolvido pela própria instituição e que objetiva, a partir de uma biblioteca dedicada de ativos virtuais representar artefatos elétricos como tomadas, eletrodutos, quadros de carga, luminárias e equipamentos de potência em uma planta bidimensional bem como realizar os cálculos referentes ao dimensionamento de condutores, disjuntores e quadro de distribuição. Paralelamente foi desenvolvido o projeto elétrico da mesma moradia no software Revit objetivando comparar o fluxo de trabalho de um projeto de edificações e a eficiência de cada ferramenta.

Inicialmente, constatou-se que uma das principais diferenças entre eles é a atribuição de parâmetros aos elementos. Enquanto no AutoCAD 2D desenham-se linhas, símbolos e outras representações geométricas para representar os componentes, distinguindo-os, principalmente, pela interpretação de legendas e notas, no Revit, por outro lado, o projetista insere diretamente itens, também denominado como famílias, com parâmetros pré-definidos nos quais são registrados características como tensão e potência.

Em virtude da utilização do Visual Electric no projeto em CAD foi possível parametrizar os elementos 2D do projeto elétrico, o que não seria possível apenas com o AutoCAD, uma vez que essa extensão permite a inserção de informações aos modelos da biblioteca atrelada à ferramenta, como pode ser observado na Figura 3.

\section{Figura 3: Projeto de Instalação Elétricas Residencial - Visual Electric - CGW}

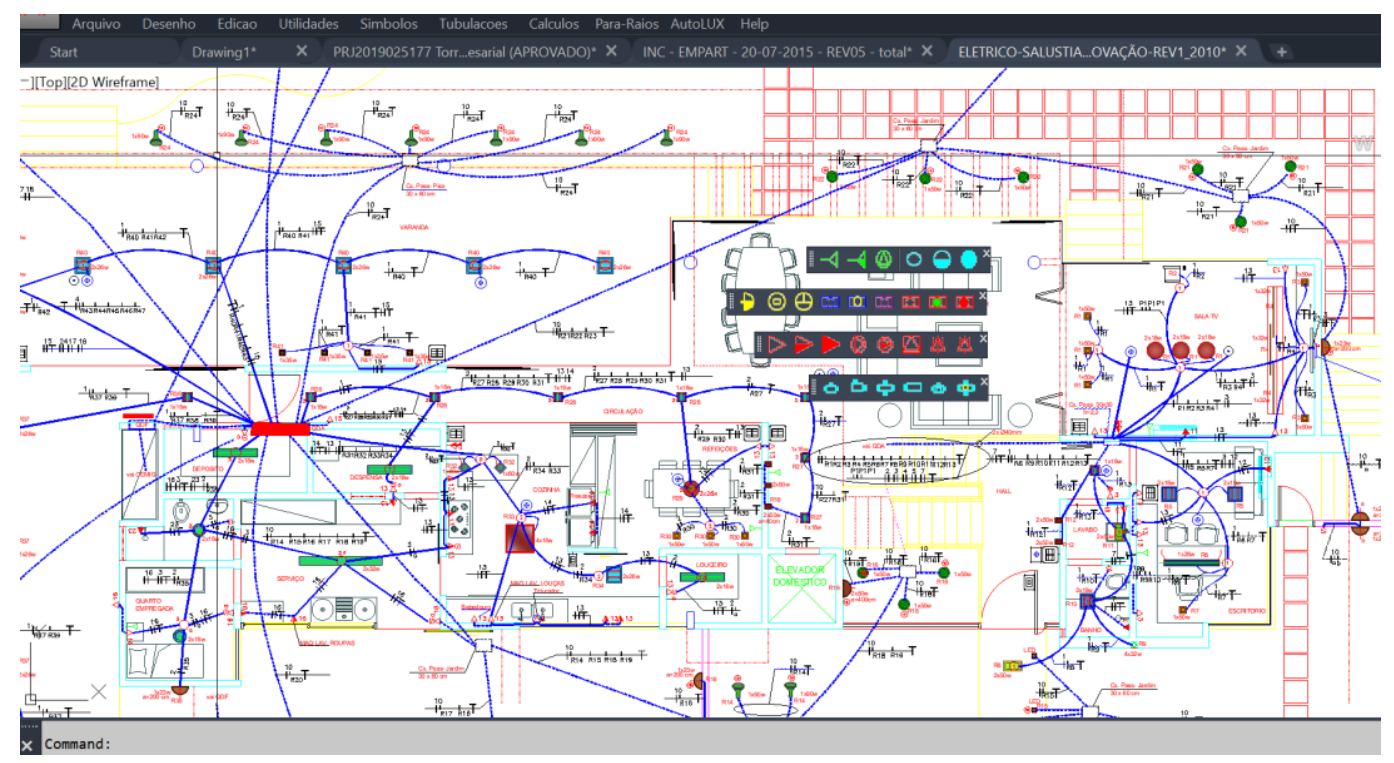

Fonte: o autor

Considerando o fato de que interferências durante a concepção de um projeto podem ser um dos primeiros impasses para a realização da compatibilização, o Revit demonstrou-se como uma alternativa eficiente e resolutiva na solução desse problema, visto que sua capacidade de automatizar a análise de interferências e os possíveis ajustes, além de separar o fluxo de trabalho das disciplinas elétrica, estrutural e arquitetônica em estruturas individualizadas e colaborativas permitiu o 
desenvolvimento síncrono de modificações e atualizações no projeto base, bem como o intercâmbio de ideias e propostas entre os projetistas ao longo do trabalho. Dessa maneira, após a importação da planta arquitetônica 2D em DWG que serviu como base para a modelagem da residência para o Revit, os lançamentos dos componentes do projeto estrutural e arquitetônico deu-se especialmente pela união em um arquivo específico RVT, possibilitando a interoperabilidade entre as ferramentas.

Em contrapartida, para identificar de maneira mais precisa as intervenções entre as disciplinas, utilizou-se a ferramenta "Verificação de interferência". Ao selecionar os componentes pertinentes para a apuração desejada, esta é orientada a apresentar e gerar um relatório de verificação apenas dos itens definidos, assegurando assim uma melhor visibilidade e eficiência no processo de verificação. Ademais, além da possibilidade de visualização global da obra aos projetistas, outra vantagem da aplicação do software BIM no processo de compatibilização é a capacidade de tornar mais precisa a representação do ambiente virtual, uma vez que os elementos adicionados são tridimensionais e podem, portanto, serem visualizados sob diversas perspectivas. Por conseguinte, tal atributo evita possíveis erros humanos no processo de planejamento e previsões futuras de integração das diversas áreas presentes na construção, possibilitando, assim, uma redução de retrabalho durante a realização dos projetos.

Paralelamente, no software AutoCAD a compatibilização é executada mediante técnicas convencionais de sobreposição e ocultação de diferentes camadas pertencentes aos projetos das disciplinas envolvidas em um arquivo CAD 2D. Assim, a análise de interferências por meio desta metodologia possui, possivelmente, limitações, uma vez que é realizada de forma manual e subjetiva, o que dificulta, normalmente, a identificação de possíveis incompatibilidades físicas e estruturais que são visualmente limitadas pela representação bidimensional.

Especificamente no projeto elétrico, desenvolvido no Revit, as conexões bifásicas e trifásicas foram realizadas de acordo com a configuração de dois ou três polos dos equipamentos, como mostra a Figura 4. Além disso, toda família que dispõe de um conector possui simultaneamente a função de conexão elétrica, o que permite atribuir todas as características elétricas essenciais para os cálculos que possam ser realizados posteriormente, o que seria, normalmente, limitado em aplicações CAD.

Por fim, após a inserção e configuração de todos os elementos no projeto, foram obtidas diversas tabelas geradas pelo software Revit, sejam elas relacionadas ao dimensionamento dos componentes, lista de materiais, detalhes da construção, orçamento, entre outros. Dessa forma, foi observado que tal funcionalidade permitiu o aumento da confiabilidade, segurança e precisão do projeto, haja vista que esses dados possibilitaram aos projetistas previsões acerca do desenvolvimento e dos recursos a serem gastos na obra. Além disso, como observado na Figura 4, o Revit possibilita a geração de quadro de cargas, cálculo luminotécnico, geração de circuitos, entre outras funções. No sistema AutoCAD, por outro lado, a geração do quadro de cargas só foi possível graças ao Visual Electric. 


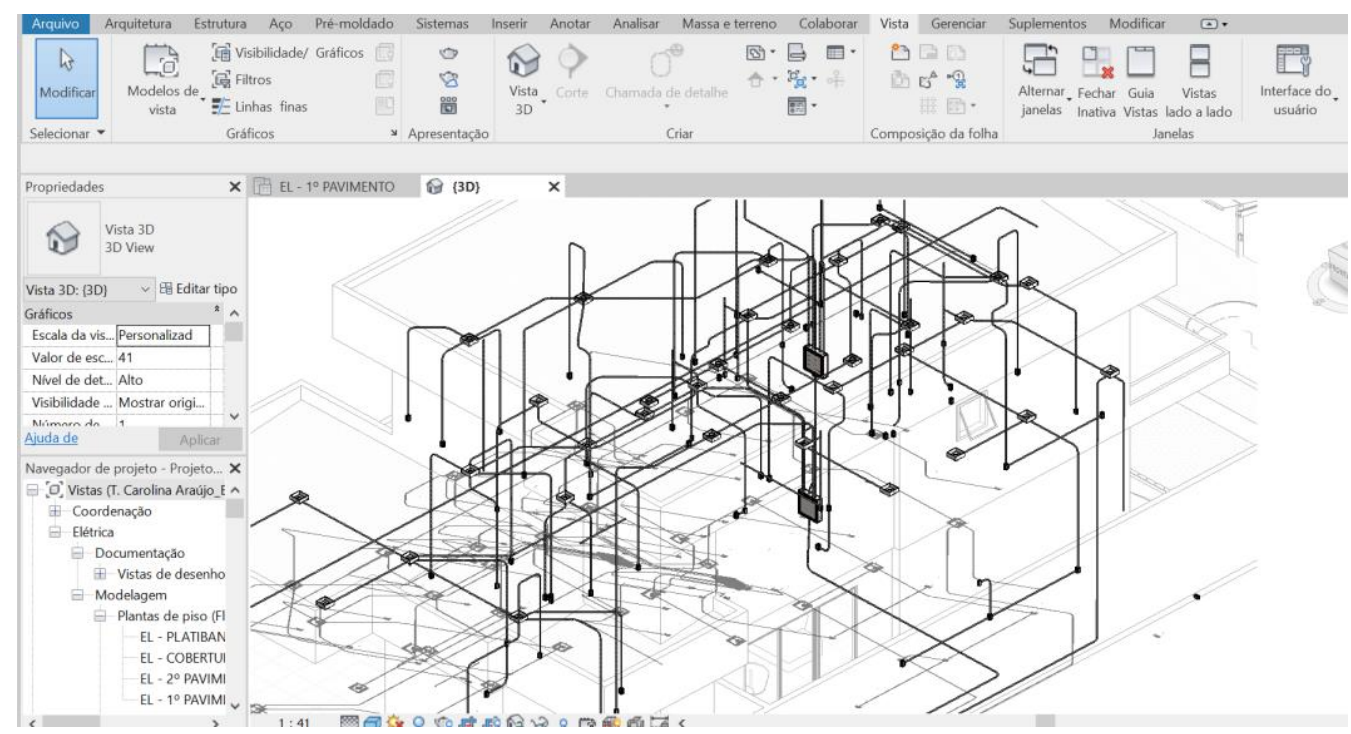

Fonte: o autor

\section{CONCLUSÃO}

No que concerne o estudo de caso tratado, por exemplo, observa-se que a capacidade de concepção síncrona da solução BIM de cada disciplina possibilitou um nível de cooperação maior ao projeto base e que o emprego, por parte da equipe, das ferramentas nativas do Revit permitiu, possivelmente, a concepção de um modelo virtual integrado, colaborativo, eficiente e mais confiável.

Pela análise realizada, considera-se normalmente que a utilização do Revit para o setor elétrico pode trazer muitas vantagens. No que tange à interoperabilidade do sistema, ou seja, a troca dinâmica de ativos virtuais e informações, sua aplicação em projetos elétricos torna-se possível a partir do software Revit, visto que houve um intercâmbio de tais modelos entre diferentes ferramentas bem como a comunicação entre os projetistas. Ademais, outro fator importante observado no estudo de caso foram as diversas funções do Revit no que se refere à visualização, como a divisão em campos de trabalho, à separação em disciplinas e à representação 3D de diversas seções e perspectivas tridimensionais possibilitaram aos usuários que as tomadas de decisão ao longo do trabalho fossem mais analisadas e discutidas, resultando, portanto, em projetos mais assertivos e com menor necessidade de retrabalho.

Todavia, tal migração requer cuidado uma vez que, caso os projetistas não apresentem as habilidades necessárias no manuseamento da ferramenta, o nível de complexidade na concepção do projeto poderá ser ainda maior em oposição a uma ferramenta CAD, fazendo com que essa não seja uma aplicabilidade eficaz.

Este trabalho objetivou, portanto, analisar as diferenças técnicas de cada software quando empregados em projetos elétricos vinculados a outras áreas do setor da construção, observando-se que, em diversas fases, a ferramenta Revit poderia ser mais completa ou eficiente que o AutoCAD. Nesse sentido, visando trabalhos futuros acerca 
do tema, deseja-se realizar novos comparativos de caráter predominantemente quantitativo no que tange às diferenças de tempo necessárias para a realização do projeto em ambas aplicações.

\section{REFERÊNCIAS}

[1] DENIZ, Gulbin Ozcan. Emerging cad and bim trends in the aec education: An analysis from students' perspective. Journal of Information Technology in Construction,[s.I.]. v. 23, p. 138-156, 2018. Disponível em: https://www.itcon.org/paper/2018/7. Acesso em: 02 ago. 2021.

[2] GCR, NIST. Cost analysis of inadequate interoperability in the US capital facilities industry. National Institute of Standards and Technology (NIST), p. 223-253, 2004.

[3] HAMMA-ADAMA, Mansur; KOUIDER, Tahar. Comparative analysis of BIM adoption efforts by developed countries as precedent for new adopter countries. Current Journal of Applied Science and Technology, p. 1-15, 2019.

[4] GERAIS, VIÇOSA-MINAS. INTEROPERABILIDADE DE SOFTWARES BIM NO CONTEXTO DE PROJETO DE ESTRUTURAS DE CONCRETO ARMADO.

[5] BRITO JUNIOR, Carlos Roberto de; TAKII, Tiago. Modelagem de projetos elétricos usando a tecnologia BIM. 2015. Trabalho de Conclusão de Curso. Universidade Tecnológica Federal do Paraná.

[6] DA COSTA, Giovani Cecatto Lopes Ribeiro; FIGUEIREDO, Sílvia Haueisen; RIBEIRO, Sidnea Eliane Campos. Estudo comparativo da tecnologia CAD com a tecnologia BIM. Revista de Ensino de Engenharia, v. 34, n. 2, 2015.

[7] NUNESA, G. H.; LEÃO, M. Estudo comparativo de ferramentas de projetos entre o CAD tradicional e a modelagem BIM Comparative study of design tools-the traditional CAD and BIM modeling. Revista de Engenharia, v. 155, n. 55, p. 47-61, 2018.

[8] DE SOUZA, Derek Santos et al. ANÁLISE COMPARATIVA DAS PLATAFORMAS BIM E CAD NA APLICAÇÃO DE PROJETOS DE EDIFICAÇÕES. Epitaya E-books, v. 1, n. 5, p. 343-358, 2020.

[9] MANAGEMENT IN CONSTRUCTION RESEARCH ASSOCIATION (MICRA) POSTGRADUATE CONFERENCE. 2012. Malaysia. Proceedings [...]. Malaysia:MICRA, 2012, p. 195-199.

[10] MENDONÇA, Kelly Roberta Moura; DE SOUSA, Pablo Gleydson; GUEDES, Emiliana de Souza Rezende. Orçamentação de obra: Análise comparativa entre metodologia tradicional e BIM. Brazilian Journal of Development, v. 6, n. 11, p. 93096-93119, 2020. 